\title{
Discrimination and diabetes: Insight from the second Diabetes Attitudes Wishes and Needs (DAWN2) Study
}

\author{
Sanjay Kalra, Manash P. Baruah ${ }^{1}$ \\ Departments of Endocrinology, Bharti Hospital and BRIDE, Karnal, Haryana, ${ }^{1}$ Excel Centre Hospitals, Guwahati, Assam, India
}

\section{A B S T R A C T}

The second diabetes attitudes wishes and needs 2 (DAWN 2) study describes various facts of living with diabetes, including barriers to, and facilitators of effective psychosocial care. One of the issues studied by DAWN2 is the phenomenon of discrimination, as viewed by persons with diabetes, family members of persons with diabetes, and health care professionals. This brief communication discusses the high prevalence of discrimination against people with diabetes, as reported by DAWN2.

Key words: Stigma, type I diabetes, type 2 diabetes, social ostracization

\section{INTRODUCTION}

Diabetes is a chronic disease, often with physical stigmata which can be easily recognized. Its impact upon daily lifestyle, diet and physical activity patterns, and at times, the need to inject frequently, create a situation where persons with diabetes appear "different" from others.

Discrimination and social stigma have been described for girls with diabetes in various countries. ${ }^{[1,2]}$ At times, the social ostracization is so severe that it impacts the very survival of girls with diabetes. Societal discrimination against adults with diabetes; however, seems to be global phenomenon, rather than one restricted to certain cultures or geographical locations.

\section{SeCond Diabetes AtTItudes Wishes AND NEEDS FINDINGS}

The recently conducted second Diabetes Attitudes Wishes and Needs (DAWN 2) study reported on views and perception of adults with diabetes, family members

\begin{tabular}{|l|l|}
\hline \multicolumn{2}{|c|}{ Access this article online } \\
\hline Quick Response Code: & Website: \\
\hline & www.josh.net \\
\hline & \\
\hline
\end{tabular}

of adults with diabetes, and diabetes care professionals from 17 countries. The 15000+ participants in DAWN 2 were chosen by a hybrid of sampling frames and recruiting methods, to reduce bias. Questions related to discrimination were posed to all three cohorts of the person with diabetes. About $17.6 \%$ persons with diabetes reported having been discriminated against because of diabetes. This proportion varied across countries (28.2\% in Turkey, 27.2\% in India, and $25.3 \%$ in Algeria, to just $10.6 \%$ in Germany and USA). This sentiment was shared by family members, who agreed that discrimination occurred against the person they lived with, because of diabetes, in $20.5 \%$ cases. Again, this response varied from $40.0 \%$ in Algeria, 35.3\% in Turkey and $31.7 \%$ in Denmark, to only $9.8 \%$ in UK, and $10.6 \%$ in the USA. Indian family members responded in the affirmative $23.4 \%$ of the times. Health care professionals too reported the need for major improvement in accepting persons with diabetes as equal members of society. While the global overage for this response was $31.1 \%$, it ranged from $78.6 \%$ in Algeria and $47.9 \%$ in India, to only $12.0 \%$ in Canada and $15.1 \%$ in UK. ${ }^{[3-5]}$

The perception of discrimination was found to be more frequent in persons on insulin therapy. Those who felt discriminated against experienced more problems living with diabetes, less perceived support, a reduced sense of well-being, and a negative impact upon relationships, work/ studies, quality-of-life, finance, leisure, and psychosocial well-being. One positive finding from DAWN 2 was that those who felt discriminated against had a higher sense 
of empowerment. ${ }^{[6]}$ Perhaps this is a defense mechanism, or a coping strategy, to deal with discrimination.

It must be remembered that DAWN 2 limited its research to adults with diabetes, and family members of adults with diabetes. It is possible that children with diabetes may face grater challenges in getting accepted by society at large.

The preliminary findings of DAWN 2 need to be studied in greater depth, but are enough to initiate action in India. In general, India has a tolerant and inclusive ethos, and every living being has a right to live. The family is expected to care for the sick and infirm. It comes as a surprise; therefore, that discrimination against diabetes is so prevalent in the country. The definition of discrimination is not a simple one, and the "perception" of discrimination is not easy to explain, either. One may take being rejected in a job interview as an example of discrimination because of diabetes. Yet another may cite having to endure a social function in which others are eating sweets and other highcalorie dishes, without regards to the sensitivity of the person with diabetes among them (culinary cruelty). Both situations are hurtful, and noninclusive.

\section{CONCLUSION}

The bitter reality of discrimination against diabetes cannot be ignored. To have an impact upon the diabetes pandemic, it needs to be fought not only at the microlevel (in the clinics and hospital wards), but also at the mesolevel, in the community and society, and at macrolevel (government and policy making).

Diabetes care professionals should expand their mandate of intervention beyond the walls of their clinics into the streets and by lanes of the community they live in. We must lead a sustained, concerted campaign to change attitudes toward diabetes, and improve empathic support provided to persons with diabetes. Involving professionals such as medical anthropologists, sociologists, health communication experts, and social marketers, will increase the quality and impact of such campaigns. Both governmental and nongovernmental organization should be included in such efforts.

\section{REFERENCES}

1. Abdoli S, Abazari P, Mardanian L. Exploring diabetes type 1-related stigma. Iran J Nurs Midwifery Res 2013;18:65-70.

2. Kalra S, Sridhar GR, Balhara YS, Sahay RK, Bantwal G, et al. National recommendations: Psychosocial management of diabetes in India. Indian J Endocr Metab 2013;17:376-95.

3. Nicolucci A, Kovacs Burns K, Holt RI, Comaschi M, Hermanns N, Ishii $\mathrm{H}$, et al. Diabetes Attitudes, Wishes and Needs second study $\left(\right.$ DAWN2 ${ }^{\mathrm{TM}}$ ): Cross-national benchmarking of diabetes-related psychosocial outcomes for people with diabetes. Diabet Med 2013;30:767-77.

4. Kovacs Burns K, Nicolucci A, Holt RI, Willaing I, Hermanns N, Kalra S, et al. Diabetes Attitudes, Wishes and Needs second study (DAWN2 ${ }^{\mathrm{TM}}$ ): Cross-national benchmarking indicators for family members living with people with diabetes. Diabet Med 2013;30:778-88.

5. Holt RI, Nicolucci A, Kovacs Burns K, Escalante M, Forbes A, Hermanns N, et al. Diabetes Attitudes, Wishes and Needs second study (DAWN2 ${ }^{\mathrm{TM}}$ ): Cross-national comparisons on barriers and resources for optimal care-Healthcare professional perspective. Diabet Med 2013;30:789-98.

6. Benedetti MM. Discrimination and diabetes. Diabetes Voice 2013;58 Suppl 2:50-3.

How to cite this article: Kalra S, Baruah MP. Discrimination and diabetes: Insight from the second Diabetes Attitudes Wishes and Needs (DAWN2) Study. J Soc Health Diabetes 2015;3:56-7.

Source of Support: Nil. Conflict of Interest: None declared. 